\title{
BI-RADS Breast Tumor Classification Through Image Mining
}

\author{
Valesca J. S. da Silva ${ }^{1,2}$, Mateus M. R. da Silva ${ }^{1}$, Marcelino P. S. Silva ${ }^{1}$, Joana R. C. Nogueira ${ }^{1}$ \\ 1 Universidade do Estado do Rio Grande do Norte \\ mateus.mendes.mmr@gmail .com \\ marcelinopereira@uern.com \\ joanarosa@uern.com \\ 2 Universidade Federal Rural do Semi-Árido \\ julianevalesca@gmail.com
}

\begin{abstract}
In this article, a computer aided diagnostic system for BI-RADS classification of breast cancer is proposed. The approach involves image processing capabilities to extract features from tumors in mammography and image mining to classify them as BI-RADS 2, BI-RADS 3, BI-RADS 4C or BI-RADS 5. Images from the BCDR repository were used for the experiments. The results showed the efficacy of the proposed method, which classified tumors with considerable accuracy in four BI-RADS categories.
\end{abstract}

Categories and Subject Descriptors: H.2.8 [Data Mining]: Database Applications; I.2.6 [Artificial Intelligence]: Learning; J.3 [Medical Information Systems]: Life and Medical Science

Keywords: breast cancer, BI-RADS, data mining, machine learning

\section{INTRODUÇÃO}

Segundo a Organização Pan-americana da Saúde, o câncer é a segunda principal causa de morte no mundo e é responsável por 9,6 milhões de mortes no ano de 2018. Em âmbito mundial, uma em cada seis mortes estão relacionadas à doença. Aproximadamente $70 \%$ das mortes por câncer ocorrem em países de baixa e média renda.

Em fevereiro de 2018, o Instituto Nacional de Câncer e o Ministério da Saúde estimaram a ocorrência de 600 mil novos casos de câncer no Brasil para esse mesmo ano. Desses 600 mil, foram esperados 59.700 novos casos de câncer de mama. O câncer de mama é a neoplasia maligna mais comum entre as mulheres no mundo e no Brasil, excetuando-se as neoplasias de pele não melanoma (Instituto Nacional de Câncer, 2019). A maneira mais eficaz de se combater essa doença ainda é a prevenção secundária, através da deteç̧ão precoce por exames de imagem, como a mamografia, pois com a descoberta precoce existe a possibilidade da realização de tratamentos que aumentam as chances de cura do paciente (Organização Pan-americana da Saúde, 2018).

A mamografia é atualmente uma das melhores técnicas de detecção precoce de lesões não palpáveis na mama, pois ela possibilita a detecção visual de alterações que podem evidenciar precocemente a presença de câncer (American Cancer Society, 2018).

O Colégio Americano de Radiologia desenvolveu o sistema Breast Image Reporting and Data System (BI-RADS) em 1992 para padronização da nomenclatura de laudos mamográficos, visando reduzir ambiguidades na interpretação de imagens da mama e recomendações de conduta, bem como facilitar o monitoramento dos resultados (Duarte, 2016).

O exame de biópsia resume-se a um procedimento cirúrgico minimamente invasivo, que visa a

Copyright(C2019 Permission to copy without fee all or part of the material printed in KDMiLe is granted provided that the copies are not made or distributed for commercial advantage, and that notice is given that copying is by permission of the Sociedade Brasileira de Computação. 
retirada de um fragmento do nódulo suspeito, para que este seja analisado. Um erro no diagnóstico (falso positivo, que pode levar à biópsia um nódulo classificado como maligno, mas que na realidade é benigno) acarreta estresse ao paciente e custo ao sistema de saúde desnecessários.

Neste sentido, o presente trabalho a princípio buscou, por meio de padrões de características das categorias do BI-RADS, o desenvolvimento de um sistema inteligente para classificação de tumores de mama em BI-RADS 2, BI-RADS 3, BI-RADS 4C e BI-RADS 5, descritos adiante.

O artigo está organizado como segue. A Seção 2 apresenta alguns trabalhos relacionados com o tema, a Seção 3 apresenta como é estruturado o padrão BI-RADS, a Seção 4 indica os procedimentos metodológicos utilizados, a Seção 5 exibe os resultados obtidos e a Seção 6 traz as considerações finais sobre o trabalho

\section{TRABALHOS RELACIONADOS}

Souto et al. (2018) desenvolveram uma metodologia e um sistema CAD para auxiliar radiologistas no diagnóstico do câncer de mama em mamografias. Foi definido um conjunto com 12 atributos morfológicos para distinguir as lesões benignas e malignas. A classificação foi realizada pelos algoritmos vizinho mais próximo (IBK), rede neural artificial Multi-layer Perceptron (MLP) e árvore de decisão Classification and Regression Trees (CART) e J48. Os algoritmos J48 e CART, obtiveram os melhores resultados, permitindo a correta classificação de $96 \%$ das mamografias, com $100 \%$ de sensibilidade e $93 \%$ de especificidade

Cruz et al. (2018) investigaram o uso de wavelets morfológicas e técnicas de aprendizado de máquina para classificar regiões suspeitas de lesão de mama em mamografias digitais, independente do tecido mamário. Os algoritmos utilizados para a classificação das lesões como normal, benigna e maligna foram o MLP, Radial Basis Function (RBF) e Support Vector Machines (SVM). O classificador MLP com taxa de aprendizado de 0,03 obteve o melhor valor de acurácia, 96,21\%.

Em Shan et al. (2016) foi realizada uma tradução completa do léxico BI-RADS de ultrassom (relacionado a forma e a borda do tumor) em recursos digitais, que são usados em métodos de aprendizado de máquina com o propósito de desenvolver um sistema CAD eficaz para o ultrassom de mama para distinguir lesões benignas de preocupantes. Foram definidos 10 atributos para diferenciar lesõs benignas de malignas. Os algoritmos utilizados para a classificação das lesões foram rede neural, árvore de decisão, SVM e floresta aleatória que obteve os melhores valores para acurácia, sensibilidade e especificidade. A rede neural obteve $78.1 \%$ de acurácia, $78.0 \%$ de sensibilidade e $78.2 \%$ de especificidade

O presente trabalho se destaca dos demais por (i) mapear as características que diferenciam as categorias de protocolo BI-RADS, (ii) adicionar e calcular recursos que indicam o padrão de ecogenicidade dos nódulos e (iii) classificar nódulos nas categorias BI-RADS 2, 3, 4C e 5, e não em benigno e maligno.

\section{BI-RADS}

Para uniformizar a comunicação da interpretação mamográfica, o Colégio Americano de Radiologia desenvolveu o sistema BI-RADS em 1992 para padronização da nomenclatura de laudos mamográficos, visando auxiliar médicos no diagnóstico quanto à probabilidade da lesão ser maligna e propondo uma conduta específica para cada caso (Geller et al., 2002).

Os médicos utilizam o padrão BI-RADS para descrever os achados e resultados da mamografia. Este padrão ordena os resultados em categorias numeradas de 0 a 6 . Ao classificar os resultados em uma dessas categorias, os médicos podem descrever o que encontram em uma mamografia usando as mesmas palavras e termos. Isto permite que a comunicação com precisão sobre os resultados e o acompanhamento após os testes sejam muito mais efetivos (American Cancer Society, 2018). O 
médico avalia o risco de malignidade da lesão e classifica o padrão mamográfico em uma das seguintes categorias BI-RADS (Duarte, 2016):

—BI-RADS 0 (incompleta): precisa de avaliação adicional por imagem e/ou mamografias prévias para comparação;

-BI-RADS 1 (negativo): não há anormalidades significativas a serem relatadas. As mamas são simétricas, sem massas, estruturas distorcidas ou calcificações suspeitas. O exame deve ser repetido anualmente ou bianualmente;

-BI-RADS 2 (benigno): este também é um resultado negativo da mamografia (não há sinal de câncer), mas o radiologista opta por descrever um achado conhecido como benigno, como calcificações benignas, linfonodos na mama ou fibroadenomas calcificados. O exame deve ser repetido anualmente ou bianualmente;

- BI-RADS 3 (provavelmente benigno): um achado avaliado usando essa categoria deve ter uma probabilidade de malignidade $\leq 2 \%$. Mas como não é comprovada a benignidade desses achados, torna-se necessário averiguar a sua estabilidade ao longo do tempo. O acompanhamento deve ser feito entre 4 a 6 meses e regularmente depois disso até pelo menos 2 anos. Essa abordagem ajuda a evitar biópsias desnecessárias. Caso haja alterações evolutivas significativas dos achados ao longo do tempo, o achado passa a ser classificado como suspeito, permitindo assim, o diagnóstico precoce. A mama pode apresentar nódulo sólido (apresentado por examente complementar de ultrassonografia) circunscrito não calcificado, assimetria focal ou agrupamento solitário de calcificações puntiformes;

-BI-RADS 4 (suspeito): esta categoria é reservada para os achados que não têm a aparência clássica de malignidade, mas são suficientemente suspeitos para justificar uma recomendação para biópsia. Esta categoria encontra-se dividida em 3 subcategorias:

- 4A (baixa suspeita): lesões com 2-10\% de chance de malignidade. A mama pode apresentar massas palpáveis sólidas e/ou massas com margens parcialmente circunscritas, cistos que necessitam de aspiração;

-4B (intermediária suspeita): lesões com 10-50\% de chance de malignidade. Pode-se encontrar massas de margens indistintas e com algumas áreas circunscritas;

— 4C (moderada suspeita): lesões com 50-95\% de chance de malignidade. Pode apresentar massas irregulares, mal-definidas ou grupamentos de calcificações pleomórficas;

-BI-RADS 5 (altamente suspeito): essas avaliações carregam uma probabilidade muito alta ( $\geq 95 \%)$ de malignidade, como massas espiculadas, de alta densidade, calcificações lineares finas e/ou massas espiculadas com calcificações pleomórficas associadas. É necessário a realização da biópsia para a confirmação;

-BI-RADS 6 (maligno): lesão com 100\% de malignidade comprovada por meio do resultado da biópsia. Lesão não retirada ou tratada.

Devido à complexidade do tema tratado, neste estágio a pesquisa está focada em 4 categorias: BIRADS 2, BI-RADS 3, BI-RADS 4C e BI-RADS 5. As demais categorias do protocolo estão sendo investigadas para posterior implementação.

\section{METODOLOGIA}

Esta seção descreve a metodologia para mineração de imagens baseada no trabalho de Souto (2014), que é constituída de duas etapas: treinamento e classificação (Figura 1):

\subsection{Treinamento}

As seguintes tarefas são executadas na etapa de treinamento: 


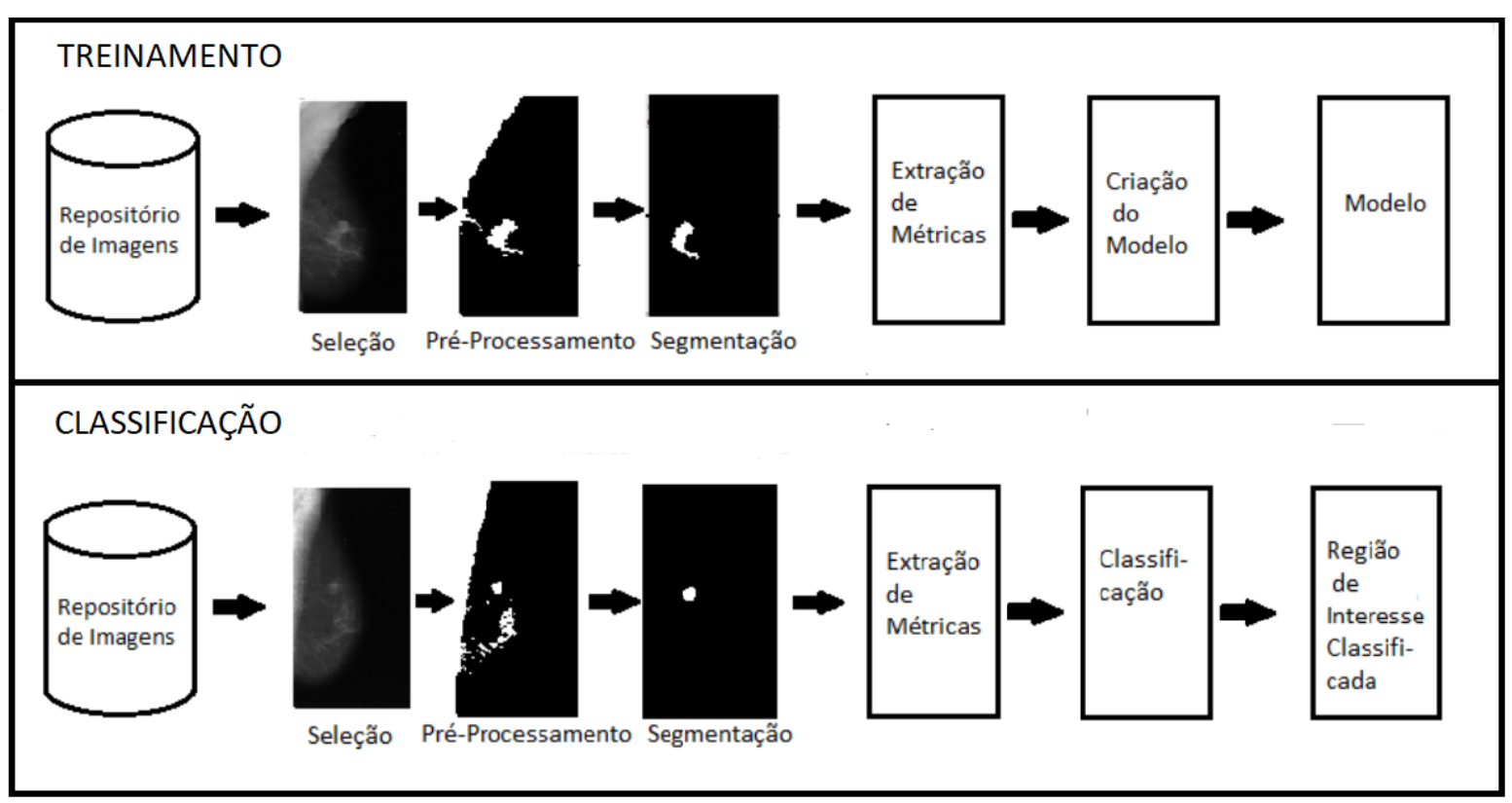

Fig. 1. Visão geral da metodologia. Souto (2014)

Seleção: a mamografia que será submetida ao processo é selecionada do repositório de imagens. $\mathrm{Na}$ etapa de treinamento as mamografias já possuem um diagnóstico;

Pré-processamento: a imagem é submetida a uma transformação. Primeiramente é aplicada a técnica CLAHE (contrast limited adaptive histogram equalization) como filtro de pré-processamento para evidenciar as lesões. Posteriormente é utilizado o filtro de limiarização, que consiste em particionar o histograma da imagem para converter todos os pixels cujo tom de cinza seja maior ou igual a um certo valor de limiar $\mathrm{T}$ em brancos e os demais em pretos. Como cada imagem possui características diferentes, é necessário que o usuário informe um limiar para cada uma;

Segmentação: nesta tarefa é aplicado o algoritmo region growing (crescimento de regiões) para segmentar a região de interesse. Este algoritmo consiste no agrupamento das regiões de acordo com a similaridade dos tons de cinza ou textura. Para este estudo a similaridade foi definida pelos tons de cinza, o filtro de pré-processamento transforme a imagem em binária;

Extração de Métricas: a partir da região de interesse segmentada, são calculadas as métricas morfológicas descritas na Tabela II;

Criação do Modelo: métricas extraídas a partir de um conjunto de ROIs (regiões de interesse, do inglês regions of interest) das imagens e seus respectivos diagnósticos são utilizados como entrada pelos algoritmos classificadores. Os algoritmos utilizados neste estudo foram SVM (kernel polinomial e grau da função do núcleo $=3$ ), K-Nearest Neighbors - KNN (com parâmetro K = 3), CART (critério de partição = índice Gini) e MLP ( 8 camadas de neurônios e taxa de aprendizado de 0.3 );

Modelo: caracteriza em padrão BI-RADS lesões que passaram pela etapa de treinamento. O modelo é utilizado na etapa de classificação.

\subsection{Classificação}

A etapa de classificação é semelhante à etapa de treinamento (Figura 1). 
Seleção: a mamografia que será submetida ao processo é selecionada no banco de imagens. Na etapa de classificação as mamografias não possuem diagnóstico;

Pré-processamento: a imagem é submetida a uma transformação. É aplicado um filtro de préprocessamento para evidenciar as lesões. São aplicados os mesmos filtros da etapa de treinamento;

Segmentação: nesta tarefa é aplicado o mesmo algoritmo da etapa de treinamento;

Extração de Métricas: a partir da região de interesse segmentada, são calculadas as métricas morfológicas descritas na Tabela II;

Classificação: as métricas extraídas são submetidas ao modelo gerado na etapa de treinamento, para que o processo de classificação indique a qual classe do BI-RADS a lesão pertence.

Região de Interesse classificada: o resultado dessa etapa é a ROI classificada como BIRADS-2, BIRADS-3, BIRADS-4C ou BIRADS-5.

O estudo feito por do Nascimento et al. (2010) com dois observadores apresentou sensibilidade (identificação de lesões malignas) que variou entre $68 \%$ e $87 \%$ além da especificidade (identificação de lesões benignas) entre $44 \%$ e $76 \%$ para mamografias. Importante resaltar que aproximadamente $15 \%$ a $30 \%$ das lesões malignas não seriam diagnosticadas e que $25 \%$ a $55 \%$ das lesões benignas seriam encaminhadas para biópsia. Diante desta margem de erro, é imprescindível a concepção de novas ferramentas que auxiliem especialistas nesta tarefa, tema do presente trabalho.

\section{EXPERIMENTOS}

O conjunto de imagens utilizadas foram extraídas do Breast Cancer Digital Repository (BCDR). O BCDR é resultado de uma colaboração entre o Centro Extremeño de Tecnologías Avanzadas (CETACIEMAT), o Instituto INEGI do Porto, a Faculdade de Medicina da Universidade do Porto e a Universidade de Aveiro. Atualmente disponibiliza dois repositórios de domínio público com imagens em níveis de cinza com 8 bits contendo: (i) filmes de mamografias digitalizadas, composto por 1010 casos de pacientes com idade entre 20 e 90 anos de idade; e (ii) mamografias digitais, com 724 casos de pacientes com idades entre 27 e 92 anos. As imagens encontram-se classificadas de acordo com o sistema BI-RADS. Além disso, também é informado o quadrante da mama que contém a lesão, o tipo (nódulo, microcalcificações, calcificações ou distorção de arquitetura, entre outros), idade e sexo do paciente, o resultado da biópsia (maligno, benigno, insuficiente ou suspeito) e a composição de tecido glandular na mama (Breast Cancer Digital Repository, 2019).

Os algoritmos foram implementados utilizando a linguagem Python e foram utilizadas as bibliotecas OpenCV para manipulação e processamento de imagens, e a Scikit-learn para mineração de dados, que possui os algoritmos mencionados na fase de criação do modelo na etapa de treinamento.

Foram selecionadas 160 imagens categorizadas de acordo com o padrão BI-RADS 2, BI-RADS 3, BI-RADS 4C e BI-RADS 5, 40 imagens de cada categoria. Para este trabalho foi elaborado um padrão de características que definem os nódulos para as categoria $2,3,4 \mathrm{C}$ e 5 do BI-RADS (Tabela I).

\begin{tabular}{|l|l|}
\multicolumn{2}{c}{ Table I. $\quad$ Características } \\
\hline BI-RADS & MORFOLOGIA \\
\hline 2 & Redondo e oval. \\
\hline 3 & Circunscrito e sólido \\
\hline $4 \mathrm{C}$ & Irregular \\
\hline 5 & Irregular e espiculado \\
\hline
\end{tabular}

Ecogenicidade é um termo usado no ultrassom que descreve quanto um tecido, órgão ou líquido deixa passar ou reflete as ondas sonoras do ultrassom em comparação com tecidos e órgãos próximos. Neste 
trabalho, estamos usando os padrões anecóico (gordura ou líquido), ecogênico (sólido) e complexo sólido-cístico (heterogêneo).

A Figura 2 apresenta exemplos de nódulos pertencentes as categorias 2, 3, 4C e 5 do BI-RADS, respectivamente. Para incluir achados na categoria 3 e 4C, precisa-se necesssariamente de exames complementares de ultrassonografia para determinar se o nódulo é sólido ou não, ou se é heterogêneo.
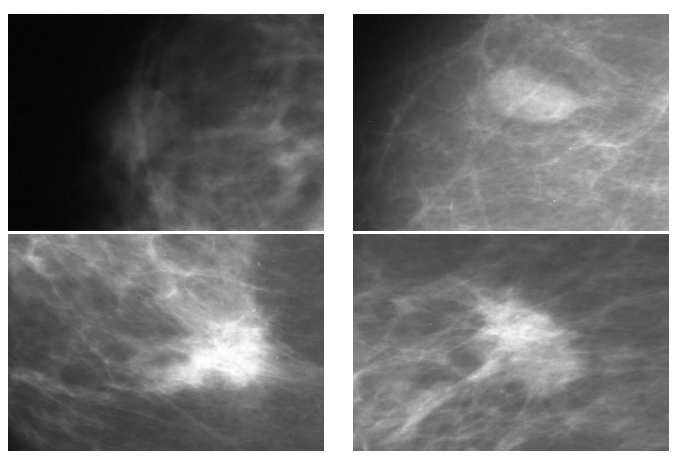

Fig. 2. Imagens mamográficas características das categorias BI-RADS 2, 3, 4C e 5, respectivamente.

Foram retirados do trabalho de Souto (2014) um conjunto de 8 atributos mais representativos e incluído o atributo solid que define se o nódulo é sólido, não sólido ou heterogêneo. Esses atributos foram usados em todos os experimentos abaixo (Tabela II).

Table II. Métricas Morfológicas

\begin{tabular}{|l|l|l|}
\hline Métrica & Equação & Descrição \\
\hline Fractal & $2 \frac{\log (0.25 * P)}{\log (A)}$ & $\begin{array}{l}\text { Índice que mede a complexidade da região. } \\
\mathrm{P}=\text { perímetro e A = área }\end{array}$ \\
\hline Max Radius & $1-\frac{A}{\pi\left(\text { raio }^{2}\right)}$ & $\begin{array}{l}\text { Retorna a distância mínima entre o centro e } \\
\text { a borda da região }\end{array}$ \\
\hline Circle & $\begin{array}{l}\text { Retorna 0 para regiões circulares e próximo de 1 } \\
\text { para regiões lineares. }\end{array}$ \\
\hline Compactness & $\left(\frac{2 \sqrt{A \pi}}{P}\right)$ & $\begin{array}{l}\text { Retorna o grau de dissimilaridade entre a região } \\
\text { e um círculo perfeito. }\end{array}$ \\
\hline Dispersion & $\frac{M a x R a d i u s}{A}$ & Mede a irregularidade de uma região. \\
\hline Shape & $\frac{P}{4 \sqrt{A}}$ & $\begin{array}{l}\text { Retorna 1 para regiões compactas e aumenta } \\
\text { de acordo com a irregularidade. }\end{array}$ \\
\hline Perimeter-Area & $\frac{P}{A}$ & $\begin{array}{l}\text { Razão entre o perímetro e a área da região. } \\
\text { É um indicador de complexidade da região. }\end{array}$ \\
\hline Spiculation & $S i=\frac{l_{i}}{b_{i}^{2}}$ & $\begin{array}{l}\text { Razão entre o comprimento da borda da região } \\
\text { e o quadrado da largura da região. Onde L é o } \\
\text { comprimento da borda da região, } \\
\text { b é o comprimento da base da região. }\end{array}$ \\
\hline Solid & $\begin{array}{l}\text { 1 Para nódulos sólidos, 0 para nódulos } \\
\text { não sólidos e 2 para nódulos heterogêneos, } \\
\text { determinados via ultrassonografia. }\end{array}$ \\
\hline
\end{tabular}

\section{RESULTADOS E DISCUSSÃO}

Para este trabalho, foram utilizadas as seguintes medidas: positivo verdadeiro, casos que a categoria BI-RADS foi classificada corretamente, falso positivo, casos em que a categoria BI-RADS foi classificada incorretamente. Para este trabalho, definimos quatro classes de positivo verdadeiro e quatro de falso positivo: VP2 (verdadeiro BI-RADS 2 positivo), VP3 (verdadeiro BI-RADS 3 positivo), VP4 
(verdadeiro positivo BI-RADS 4C), VP5 ( BI-RADS 5 positivo verdadeiro), FP2 (BI-RADS 2 falso positivo), FP3 (BI-RADS 3 falso positivo), FP4 (BI-RADS 4C falso positivo) e FP5 (BI-RADS 5 falso positivo). A métrica de avaliação utilizada foi a acurrácia, que corresponde à taxa de exemplos corretamente classificados.

A técnica CLAHE foi aplicada às imagens e, em seguida, a técnica de limiarização, onde o limiar para cada imagem Ãl' dado manualmente. A segmentação foi realizada e o conjunto de características foi extraído. A entrada para os algoritmos de aprendizado de máquina (CART, KNN, SVM e MLP) é um arquivo .csv que contém as características extraídas após a segmentação para criar o modelo de treinamento e executar a classificação do ROI em 4 classes (BI-RADS 2, 3, 4C e 5).

A avaliação da eficiência da representação dos dados proposta neste trabalho foi realizada em várias sessões de treinamento e classificação usando validação cruzada. Os dados foram divididos em 10 conjuntos com 16 imagens, sendo 4 imagens de cada categoria do BIRADS. A Tabela III mostra o resultado da classificação das imagens com a validação cruzada. O algoritmo CART obteve o melhor resultado com acurácia de 93,12\%, seguido pelo algoritmo SVM com 82,50\%, MLP com 68,75\% e KNN com $66,87 \%$. O valor de acurária é a média dos acertos de cada etapa da validação cruzada.

Table III.
\[ \text { Resultado da Validação Cruzada } \]
\begin{tabular}{|l|l|}
\hline Algoritmo & Acurácia \\
\hline CART & $93,12 \%$ \\
\hline KNN & $66,87 \%$ \\
\hline SVM & $82,50 \%$ \\
\hline MLP & $68,75 \%$ \\
\hline
\end{tabular}

O gráfico na Figura 7 indica a acurácia dos métodos de classificação em cada uma das categorias BIRADS. O algoritmo SVM classificou 95\% das mamografias BI-RADS 2 corretamente. Os algoritmos CART e SVM classificaram corretamente $100 \%$ dos mamogramas BI-RADS 3. O algoritmo CART classificou corretamente 100\% das mamografias BI-RADS 4C. O algoritmo CART classificou 87,5\% das mamografias BIRADS 5 .

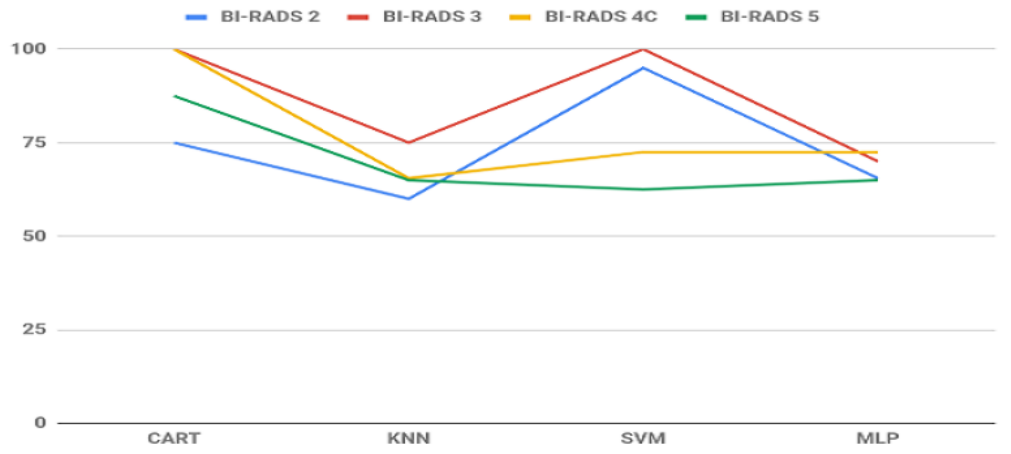

Fig. 3. Acurácia dos métodos de classificação em cada categoria do BI-RADS . Souto (2014)

\section{CONSIDERAÇÕES FINAIS}

Neste estudo, propusemos o desenvolvimento das funções iniciais de um sistema inteligente para mineração de imagens mamográficas a fim de classificar nódulos mamários utilizando o protocolo BI-RADS. O sistema tem como objetivo auxiliar os radiologistas, fornecendo uma segunda opinião sobre a análise de lesões em mamografias. A diferença deste estudo em relação aos esquemas CAD anteriores, inclui (i) mapear as características que diferenciam as categorias de protocolo BI-RADS, 
(ii) adicionar e calcular recursos que indicam o padrão de ecogenicidade dos nódulos e (iii) classificar nódulos nas categorias BI-RADS 2, 3 4C e 5 e não em benigno e maligno.

Como o padrão de ecogenicidade é obtido por imagens de ultrassom da mama, neste primeiro momento, essas informações são fornecidas pelo usuário. No futuro, pretendemos obter imagens de ultrassonografia mamária que complementem as mamografias. Com isso, podemos extrair mais recursos que complementam o padrão de cada categoria BI-RADS e tornar o sistema mais robusto.

Como trabalhos futuros pretende-se incluir as categorias 4A e 4B do BI-RADS. Também pretendese refazer os testes com mamografias digitais para verificar se haverá diferença significativa. Novos métodos de processamento de imagem, algoritmos de segmentação e outros métodos de aprendizado de máquina também devem ser incorporados à proposta.

\section{REFERENCES}

American Cancer Society. About breast cancer. https://www.cancer.org/cancer/breastcancer/about/what-is-breast-cancer.html, 2018. Data de acesso: 6 fev. 2019.

Breast Cancer Digital Repository. More about bcdr. https://bcdr.eu/information/about, 2019. Data de acesso: 13 fev. 2019.

Cruz, T., Cruz, T., and Santos, W. Detection and classification of lesions in mammographies using neural networks and morphological wavelets. IEEE Latin America Transactions 16 (3): 926932, 2018.

do Nascimento, J. H. R., DA Silva, V. D., And Maciel, A. C. Acurácia dos achados mamográficos do câncer de mama: correlação da classificação BI-RADS e achados histológicos. Radiologia Brasileira 43 (2): 91-96, 2010.

DuARTe, D. ACR BI-RADS: sistema de laudos e registro de dados de imagem da mama: Atlas de diagnóstico por imagem da mama. São Paulo: Colégio Brasileiro de Radiologia vol. 2, pp. 1-574, 2016.

Geller, B. M., Barlow, W. E., Ballard-Barbash, R., Ernster, V. L., Yankaskas, B. C., Sickles, E. A., Carney, P. A., Dignan, M. B., Rosenberg, R. D., Urban, N., et al. Use of the american college of radiology bi-rads to report on the mammographic evaluation of women with signs and symptoms of breast disease. Radiology 222 (2): 536-542, 2002.

Instituto NACIONAL DE CÂNCER. Conceito e magnitude do câncer de mama. https://www.inca.gov.br/controle-do-cancer-de-mama/conceito-e-magnitude, 2019. Data de acesso: 6 fev. 2019.

OrganizaÇÃo PAn-Americana da SAúde. Folha informativa: Câncer. https://www.paho.org/bra.../index.php?option=comcontentview $=$ articleid $=5588$ :folhainformativa-cancerItemid $=1094,2018$. Data de acesso: 8 fev. 2019.

Shan, J., Alam, S. K., Garra, B., Zhang, Y., and Ahmed, T. Computer-aided diagnosis for breast ultrasound using computerized bi-rads features and machine learning methods. Ultrasound in medicine 83 biology 42 (4): 980-988, 2016.

Souto, L. P. M. Mineração de imagens para a classificação de tumores de mama. M.S. thesis, Universidade do Estado do Rio Grande do Norte. Universidade Federal Rural do Semi-Árido., 2014.

Souto, L. P. M., Dos Santos, T. K., And Silva, M. P. S. Classification of breast tumors through image mining techniques. In Anais do XVIII Simpósio Brasileiro de Computação Aplicada à Saúde. SBC, 2018. 